\title{
LONGITUDINAL EVALUATION OF CEREBRAL FUNCTION THROUGH PROTON MAGNETIC RESONANCE SPECTROSCOPY IN SYSTEMIC LUPUS ERYTHEMATOSUS
}

Renan Bazuco Frittoli (Unicamp, Mogi Mirim, SP, Brasil), Danilo Rodrigues Pereira (Unicamp, Limeira, SP, Brasil), Mariana Postal (Unicamp, Campinas, SP, Brasil), Leticia Rittner (Unicamp, Campinas, SP, Brasil), Lilian Tereza Lavras Costallat (Unicamp, Campinas, SP, Brasil), Simone Appenzeller (Unicamp, Campinas, $\mathrm{SP}$, Brasil)

\section{BACKGROUND}

Neuropsychiatric impairment in Systemic Lupus Erythematosus (SLE) has gained focus and importance. Proton magnetic resonance spectroscopy ( $\left.{ }^{1} \mathrm{H}-\mathrm{MRS}\right)$ has been shown to be an important non-invasive tool to quantify neuronal damage in central nervous system disorders. The objective was to analyze during a period of $9.3 \pm 7.1$ months, the presence of axonal dysfunction in SLE and to determine clinical, laboratory and treatment features associated with its occurrence and to associate axonal dysfunction with sera Th1, Th2 and Th17 cytokines levels.

\section{MATERIALS AND METHODS}

We included 203 consecutive SLE patients from the Rheumatology outpatient unit and 247 healthy controls. All patients underwent two magnetic resonance imaging (MRI) exams. We performed multi voxel $1 \mathrm{H}-\mathrm{MRS}$ using point resolved spectroscopy sequence over the superior-posterior region of the corpus callosum (3T Phillips ${ }^{\circledR}$ scanner) and signals from $\mathrm{N}$-acetylaspartate (NAA), choline (Cho); creatine ( $\mathrm{Cr}$ ), glutamate (Glu), glutamine (Gln) and lactate (Lac). A complete clinical, laboratory and neurological evaluation was performed in all subjects. Neurological manifestations were analyzed according to the ACR classification criteria. Mood and anxiety disorders were determined through Beck Depression (BDI) and Beck Anxiety Inventory (BAI). SLE patients were further assessed for clinical and laboratory SLE manifestations, disease activity [SLE Disease Activity Index (SLEDAI)], damage [Systemic Lupus International Collaborating Clinics/American College of Rheumatology Damage Index (SDI)] and current drug exposures. Th1 (IL-12, TNF- $\alpha$, IFN- $\gamma$ ), Th2 (IL-6 and IL-10), Th17 (IL-17) cytokines levels were measured by ELISA

\section{RESULTS}

NAA $/ \mathrm{Cr}(p=0.002)$ were significantly decreased and $\mathrm{Cho} / \mathrm{Cr}(p=0.045)$ and $\mathrm{Gln} / \mathrm{Cr}$ levels $(p=0.05)$ was increased in SLE patients when compared to healthy controls. When compared SLE in two times of ${ }^{1} \mathrm{H}$ MRS, 50.4\% SLE patients presented reduction of levels of NAA/Cr and $44.8 \%$ SLE patients presented increased of levels of Cho/Cr. We observed that NAA/Cr was associated with presence of symptoms of anxiety $(p=0.032)$ and correlated with levels of BAI $(r=-0.005, p=0.05)$ and TNF- $\alpha(r=0.181 ; p=0.004)$. Cho/Cr was associated with aDNAn $(p=0.028)$ and correlated with TNF- $\alpha(r=0.019 ; p=0.002) . \mathrm{G} \ln / \mathrm{Cr}$ associated with aDNAn ( $p=0.02)$ and presence of ANA (0.035). Glu/Cr ratio correlated with levels of BAI $(r=0.120, p=0.033)$, levels of BDI $(r=0.30, p=0.03)$ and IL-6 levels $(r=-0.115, p=0.05)$.

\section{CONCLUSION}

We observed significant axonal dysfunction in SLE. Decreased NAA/Cr ratio was associated with symptoms of anxiety and correlated with TNF- $\alpha$. Increased Cho/Cr ratio was associated with aDNAn and correlated with TNF- $\alpha$. Glu/Cr correlated with anxiety levels of BAI, depression levels of BDI and IL-6 levels, suggesting brain injury. 